\title{
Wisdom about the Crowd: Assuring Geospatial Data Quality Collected in Location-Based Games
}

\author{
Sebastian Matyas ${ }^{1}$, Peter Kiefer ${ }^{1}$, Christoph Schlieder ${ }^{1}$, and Sara Kleyer ${ }^{2}$ \\ Otto-Friedrich University Bamberg \\ ${ }^{1}$ Chair for Computing in the Cultural Sciences, \\ ${ }^{2}$ Chair of Statistics and Econometrics, \\ Feldkirchenstrasse 21, 96047 Bamberg, Germany \\ \{sebastian.matyas, peter.kiefer, \\ christoph.schlieder, sara.kleyer\}@uni-bamberg.de
}

\begin{abstract}
The idea of outsourcing geospatial data creation tasks to the crowd (volunteered geographic information, VGI) has become quite popular in the field of geographic information science (GIScience). As one approach to VGI, location-based games (LBGs) have been shown to be successful in motivating non-expert users to collect and tag geospatial data. However, the central VGI problem of data quality has so far not been solved satisfyingly. Previous studies show that games that reward their players for in- or post-game data reviewing can assure only a validation rate of about $40 \%$ of the data. We address this problem with a new LBG design pattern, based on game rules that encourage players to take the decisions of others into account while making their data collecting decisions. We empirically evaluate the new pattern by comparing the positional accuracy of data collected with two different rule sets for the LBG GeoSnake. Our pattern is shown to result in a significant accuracy improvement.
\end{abstract}

Keywords: location-based games, crowdsourcing, volunteered geographic information, geospatial data, data quality, game design.

\section{Introduction}

Ahn and Dabbish (2008) [1] have shown that regular internet users can be motivated by games to work on simple tasks creating outputs that are useful for serious applications, like search engines, without any kind of incentive other than entertainment. Using web-based games like the ESP game (see [1] or http://www.espgame.org/gwap/) they were able to gather huge amounts of data, in the referenced case semantic tags for images. Projects, such as OpenStreetMaps (www.openstreetmap.de/), have picked up this idea for the domain of geospatial data and count on enthusiastic amateurs to map the world for free.

Matyas et al. (2009) [8] have demonstrated that Ahn's idea can be adopted for the creation of geospatial data with the help of location-based games (LBG). But they reported that, unlike in a pure web-based context, volunteers of LBGs are rather reluctant to review the data created by other players. 
The main contributions of this paper therefore are: We first propose an approach to assure the geospatial data positional accuracy collected in a LBG, without introducing a special review board like in [5] for example. It is inspired by the idea of the wisdom of the crowd [9] and realized as a game design pattern, in the spirit of Björk and Holopainen's [3], that can be integrated into any geospatial data collecting LBG. Instead of adding an ex-post quality assurance process it succeeds in "persuading" players to create accurate data by taking into account the decisions of others while making their data collecting decisions.

As a second contribution, this paper empirically evaluates the new game pattern and shows that it results in an improvement of positional accuracy. A study was performed in an experimental setting with the location-based game GeoSnake.

The rest of the paper is structured as follows: in the next section, we will give a short overview on related work. Section 3 introduces the wisdom-about-the-crowd pattern and the location-based game GeoSnake. The game has been used in an experimental setting in a case study (subsection 3.1 and 3.2). We summarize our findings in section 4 .

\section{Related Work}

The usage of LBGs to gather geospatial data has been discussed in the game research community for some years. Capra et al. (2005) [4] were among the first to mention this possibility. Since then, various LBGs have been introduced that allowed the collection of various types of geospatial data as a by-product of game play.

CityExplorer. [8] was the first LBG designed with the primary goal to collect geospatial information. In CityExplorer players create complex geospatial data sets, composed of two GPS coordinate pairs, image data, and a semantic tag.

The central problem that remains open for all LBGs so far (see also [8] for an overview) is how to guarantee the quality of the collected data sufficiently well. So far either none (e.g. [6]), or a point-based review system (e.g. [2, 5, 8]) is used to validate the correctness of player-created data. Interestingly, e.g. [2, 5, 8] reported nearly the same results of how much data was actually reviewed by the players of their games. The review rate lies always between $30 \%$ to around $40 \%$. Though one can argue whether these studies constitute a representative sample, they give a strong hint that the upper bound for the quota of data that gets checked with a review system is indeed around $40 \%$. Thus, more than half of the collected data can be regarded as of unknown quality. While the quality indicators of the hardware (e.g., a GPS device) could be known and used as measures, the players could still have provided erroneous data on purpose. So the review system also serves the role of an anti-cheating tool [8] and cannot be replaced so easily by hardware characteristics alone. The game design pattern presented in the following section therefore strives to assure quality and fairness at the same time.

\section{Wisdom about the Crowd Gaming: GeoSnake Study}

To overcome the above mentioned limitations of a game-based review system we propose a new kind of quality assurance strategy for LBGs. It is important to note that the 
positional accuracy is not primarily affected by the ability of the player to handle a GPS receiver and his or her knowledge about geodetic datums and map projections. Almost all issues requiring technical background knowledge are handled by the game software. The major problem associated with recording the position of a geographic object relates to semantic ambiguities. A building may have several entrances - which of them should be used to mark the position of the building? There are even more alternatives for extended objects such as streets or regions. For example a query on Google Maps for the "OttoFriedrich University of Bamberg" returns the coordinate pair 49.90763,10.90466. But the same query on Bing Maps results in 49,89378,10,88595. Single expert data collectors have obviously problems to solve the semantic ambiguity problem, so how should nontrained volunteers have a chance to solve it? Clustering multiple recordings from different volunteers also doesn't solve the problem as the cluster center will certainly end up somewhere without a semantic meaning to the real-world place.

With this question in mind we propose the following general game design pattern:

\section{Wisdom about the crowd}

Description: Players are retained to take into account the anonymous majority decision of the other players when generating game-based geospatial data.

Consequences: The pattern is tailored to provide a kind of implicit quality control mechanism for geospatial data creation LBGs. However, we do not expect the crowd to outperform experts with respect to quality - a claim associated with the original wisdom of the crowd idea by [9]. Also, the basic mechanism is different from that of typical wisdom of the crowd approaches. It mimics a review strategy by trying to motivate the players to create data that most of the players can agree on, not with an external reward (or punishment) system but by a specifically designed game rule. The players are "lured" to provide agreeable data on their own. It thus goes beyond the traditional reviewing system, such as the peer reviewing of [5] or [8], as the data is after all indirectly evaluated by all players and not just a few players that happen to be on the review board. Note that the pattern is somehow similar to the approach used in [7] but uses an anonymous crowd as the "opposing" player and not just a single one.

\subsection{Set-Up}

We will demonstrate the usage of the pattern with the introduction of a simple LBG, GeoSnake - a variant of the popular video game Snake. Like the video game, GeoSnake is a single-player game. In contrast to the genuine, players have to visit a known number of places and choose appropriate GPS coordinates for them. Players get points for every place visited. Points are deducted if a player crosses his previous path (one point) or takes a path twice (two points) - so every street can be seen as a one-way street and the tail of the snake is growing alongside the path the player takes continuously. Now this might appear easily done, in a real world city wide game field it is quite a challenge. Please not that for a non-experimental setting a multiplayer game would be more appropriate as a design for a location-based geospatial data gathering game (see [8] on this subject). 
For GeoSnake, we formulated the following two game rules for how the players should pick a GPS coordinate for a place in the game. The first one is the normal rule that one would expect as a result from the game description we gave above: Choose a GPS coordinate that identifies the place without a doubt, so located inside or as near as possible to the place (called V1). The second one represents the implementation of the wisdom-about-the-crowd game design pattern: Choose a GPS coordinate, that you think the other players also chose (called V2). For V1 points were awarded as long the chosen GPS coordinate was reasonable near the place in question. For V2 only those players received points whose coordinate pair belonged to the biggest cluster of coordinates for a given place.

The hypothesis is that the players will produce more accurate geospatial data under rule variation $\mathrm{V} 2$ than under variation $\mathrm{V} 1$.

To judge the accuracy and therefore the quality of the provided geospatial data we proceed as follows with the GPS coordinates of V1 and V2: (1) We compute the individual cluster centers for all places used in the game and then (2) we measure all distances between the cluster centers and the associated GPS coordinates for a place. So we end up with a table that holds all distances for a GPS coordinate to its associated cluster center for both rule variants. To evaluate the effect of the employed rules a generalized linear model (GLM) is estimated:

$$
\mu=E(\text { distance|rule, } \text { time })=h\left(\beta_{0}+\beta_{1} \text { rule }+\beta_{2} \text { time }\right)
$$

GeoSnake was used in the case study to test the hypothesis provided above, to control for learning effect after rule switching. Therefore, the case study has a randomized treatment selection. GeoSnake was played by a group of 15 students and employees of the university of Bamberg with a background in either computer science (4) or humanities (11). Gender was mixed with 5 male and 10 female participants, age mean was 26,6 years.

To be sure to collect the same amount of GPS coordinates under each rule (V1 and V2) we had two game fields each with six places for the players to choose GPS coordinates for (1V and $2 \mathrm{~V})$. We then used a pseudo-random allocation of player, rule and place set. So each player actually had to play two rounds of GeoSnake in course of the case study, for e.g. the first participant played with the places of $1 \mathrm{~V}$ with $\mathrm{V} 1$ and then played on $2 \mathrm{~V}$ using $\mathrm{V} 2$. Then we used $2 \mathrm{~V}$ under $\mathrm{V} 1$ and $2 \mathrm{~V}$ under $\mathrm{V} 2$, etc.

\subsection{Results}

For the reason of estimating a GLM with canonical link of a Gamma distributed response with the $R$ software, the directions of the effects have to be interpreted oppositely in table 1 as $R$ reports the inverse as response. Using the above explained GLM (equation 1) the results show that rule 2 is better than rule 1 on a significance level of 0.05 . See table 1 for the result chart from $R$.

Table 1. Source: GeoSnake Game. Remarks: $* * *<0 ; * * 0.05 ; *<0.1$

\begin{tabular}{lll}
\hline & Coefficient & Std. Error \\
\hline rule 1 (Intercept) & $0.014 * * *$ & 0.002 \\
rule 2 & $0.008 * *$ & 0.003 \\
time & $-0,0002$ & 0.0002 \\
\hline
\end{tabular}


To illustrate this finding figure 1 pictures two examples of data sets, one for a street and one for a building from the places of the game field $2 \mathrm{~V}$. The red dots show GPS coordinates chosen using rule V1 and the blue dots using rule V2. It is easy to see that rule V2 produced a much more denser cluster of GPS coordinates than rule V1.
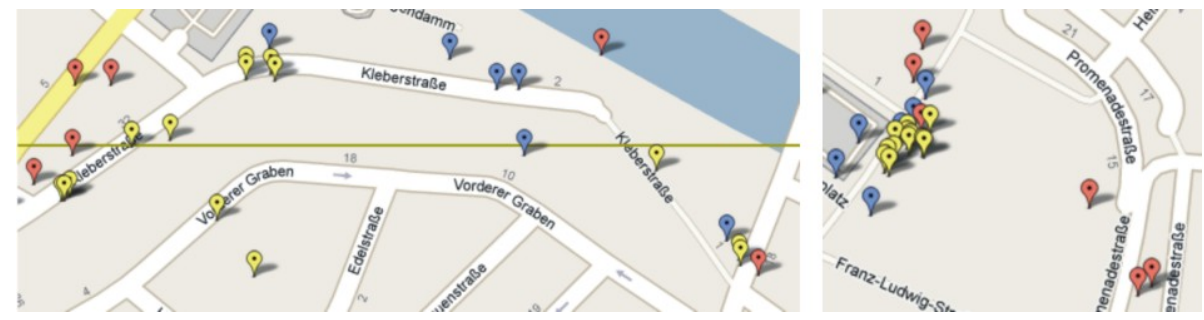

Fig. 1. Visualization of the chosen GPS coordinates for two GeoSnake places, a street (left image) and a building (right image) on game field $2 \mathrm{~V}$

Note that the building - right image of figure 1 - can be accessed from two sides, the entrance point on the left - where all blue dots are placed - is the main entrance. The right entrance, chosen by three players under V1 (red dots), is a secondary entrance where one can enter the building coming from the nearby bus station. Players under V1 obviously didn't care that much about the quality of the resulting geospatial data but more about the game itself - not crossing their way later in the game for example. Under V2 it seems that it is just the other way around or put differently that our implemented game pattern made them more aware of the underlying semantic ambiguity problem as discussed at the beginning of this section.

The street example (left image of figure 1) supports this impression. Here the blue dots are centered around the middle of the street - after the game several players reported that there is a well-known coffee shop located. The players choosing the red dots were actually more concerned that when they entered the street that they had to go a long way to avoid taking that way twice or crossing it. So they took the most favorable decision from a game play perspective.

These examples demonstrate how the implementation of the wisdom-about-thecrowd pattern into the GeoSnake game "tricks" players into providing geospatial data that the majority of the other players can agree on. Furthermore it prohibits that the players only think about the game when creating the geospatial data but also about the quality of the data with regard to the semantic ambiguity problem.

\section{Conclusions}

In this paper we have shown that the $40 \%$ participation rate that is common for review systems of geospatial data creation location-based games to date can be overcome by implementing the wisdom-about-the-crowd design pattern. We presented the general design pattern and the location-based game GeoSnake to illustrate its use. Additional we used our game in the course of a case study to validate the hypothesis that with our 
proposed game pattern the spatial accuracy of the collected data can be increased. Open interviews and detailed data analysis point out that these results are independent of the point-based game rewards used in GeoSnake. Related work suggests that when geospatial data creation is paired with a location-based game, players are more concerned about the game and not so much about qualitative data creation. The results from the GeoSnake use case study indicate that this behavior changes when our proposed design pattern is applied to such a game.

\section{References}

1. von Ahn, L., Dabbish, L.: Designing games with a purpose. Commun. ACM 51(8), 58-67 (2008)

2. Bell, M., Reeves, S., Brown, B., Sherwood, S., MacMillan, D., Ferguson, J., Chalmers, M.: Eyespy: supporting navigation through play. In: CHI, pp. 123-132. ACM, New York (2009)

3. Björk, S., Holopainen, J.: Patterns in Game Design. Charles River Media, Inc., Rockland (2004)

4. Capra, M., Radenkovic, M., Benford, S., Oppermann, L., Drozd, A., Flintham, M.: The multimedia challenges raised by pervasive games. In: International Conference on Multimedia, MULTIMEDIA 2005, pp. 89-95. ACM Press, New York (2005)

5. Grant, L., Daanen, H., Benford, S., Hampshire, A., Drozd, A., Greenhalgh, C.: Mobimissions: the game of missions for mobile phones. In: SIGGRAPH 2007: ACM SIGGRAPH 2007 Educators Program, p. 12. ACM, New York (2007)

6. Drozd, A., Benford, S., Tandavanitj, N., Wright, M., Chamberlain, A.: Hitchers: Designing for cellular positioning. In: Dourish, P., Friday, A. (eds.) UbiComp 2006. LNCS, vol. 4206, pp. 279-296. Springer, Heidelberg (2006)

7. Hacker, S., von Ahn, L.: Matchin: Eliciting user preferences with an online game. In: CHI 2009, pp. 1207-1216. ACM, New York (2009) 978-1-60558-247-4/09/04

8. Matyas, S., Matyas, C., Mitarai, H., Kamata, M., Kiefer, P., Schlieder, C.: Designing location-based mobile games - the cityexplorer use case. In: Digital Cityscapes: Merging Digital and Urban Playspaces, pp. 187-203. Peter Lang Publishers (2009)

9. Surowiecki, J.: The wisdom of crowds. Doubleday, New York (2004) 